Neuropeptides 6: $527-530,1985$

\title{
ENDOGENOUS OPIOIDS ARE INVOLVED IN ABNORMAL STEREOTYPED BEHAV- IOURS OF TETHERED SOWS
}

Gregory M. Cronin, Piet R. Wiepkema and Jan M. van Ree

Ethology Section, Department of Animal Husbandry, Agricultural University, Postbox 338, 6700 AH Wageningen, The Netherlands

* Rudolf Magnus Institute for Pharmacology, Medical Faculty, University of Utrecht, Vondellaan 6, 3521 GD Utrecht, The Netherlands

(Reprint requests to JMVR)

\section{ABSTRACT}

Tethered sows continuously performed stereotypies during a substantial part of the day time. A single subcutaneous injection with naloxone significantly decreased the profound stereotypies, while explorative behaviours of pigs were not affected by naloxone. In addition a long-term effect of naloxone was noticed. It is suggested that performing the seemingly purposeless stereotypies may function as an effective strategy in order to cope with the conflict-inducing housing conditions. The present data implicate endorphins in this strategy. The involvement of endorphuns in the stereotypies of the sows may be related to the calming, rewarding and dependency-inducing effects of the endorphins.

\section{INTRODUCTION}

Abnormal stereotyped behaviours (stereotypies) have long been noticed in pet shop, circus and zoo-animals but recently also in certain domestic farm animal species subjected to modern intensive techniques of animal housing. Among these are breeding sows subjected to individual housing and tethering. When sows are initially singly housed and neck tethered, they perform escape attempts, aggressive acts against the environment and other behaviours, which can be described as "hiding", e.g. pressing their snouts into the corner of the trough for long periods. After some hours the sows quieten down, and may remain so for a period of time up to a few weeks, although they still show episodes of "hiding". Then, sows will perform short bursts of multiple repetitive behaviours, which are often aggressivelike. Subsequently, there is a selection for one particular pattern of behaviour, performed in a repetitive manner with longer bout lengths and which may be considered as toned-down aggressiveness. Initially, these stereotyped behaviours are only present in the periods following feeding, but at a later stage (5-8 weeks after tethering) the sows continuously perform stereotypies during a substantial part of the day time (1). The stereotypies vary among the individual sows, but are rather stable for each sow both qualitatively and quantitatively.

The function of the profound and long-lasting stereotypies is obscure. Maybe by performing these behaviours the sows are calmed and succeed in coping with their aggression and the conflict induced by the environment. Consequently, sows may gain reward from their stereotyples, Noreover, the continuous performance 
of these behaviours resembles to a certain extent self-stimulation and addictive behaviours. Since endorphins and especially $\beta$-endorphin have been implicated in the physiological processes underlying reward, and may be involved in both self-stimulation and addictive behaviours (2), the postulate emerged that the stereotypies of the sows are related to the action of endorphins. To test this hypothesis the effect of the specific opioid antagonist naloxone was explored in tethered sow's showing profound stereotypies.

\section{OUTLINE AND RESULTS OF EXPERIMENTS}

Eight tethered sows which had performed unchanged stereotypies for 4 to 64 weeks entered the study. They were accustomed to being neck tethered and to single housing. Feeding occurred at $8.45 \mathrm{a} . \mathrm{m}$. and $3.30 \mathrm{p.m}$., while water was available ad libitum. Most sows had access to an "extra" chain, which was suspended in the front of their stall. Continuous behaviour observations were recorded either directly by an observer or indirectly via video-equipment. The duration, frequency and sequence of the individual behaviour elements were measured, at least between 9.30 and $11.30 \mathrm{~h}$ on treatment days. Eight classes of behaviour were distinguished (Table 1). In most sows, the normally occurring stereotypies were chain-related behaviours, but some also involved sham actions and tether chain chewing (see 1 for a description of typical sow stereotypies). The sows were subcutaneously injected with placebo (salinc) or naloxone $(0.7-1 \mathrm{mg} / \mathrm{kg}$ ) at $9.30 \mathrm{a} . \mathrm{m}$. Half of the sows received on subsequent days placebo, naloxone, placebo as treatment regime, while the others received placebo, placebo, naloxone. At the time of injection , on treatment days, the sows had performed their stereotypies for at least $30 \mathrm{~min}$.

Repeated placebo injections hardly changed the stereotypies of the anirnals. However, naloxone treatments resulted in a marked decrease in the performance of the normally occurring stereotypies (Table 1; an example is presented in figure 1).

\section{TABLE 1}

Effect of naloxone on the various behaviours performed by tethered sows. The values shown in the table are the median proportions of time that the sows performed the indicated behaviours during the first two hours after treatment.

\begin{tabular}{|c|c|c|c|c|c|c|c|c|}
\hline Class of behaviour ${ }^{1}$ & 1 & 2 & 3 & 4 & 5 & 6 & 7 & 8 \\
\hline Placebo treatment ${ }^{2}$ & 86 & 0 & 0 & 3.5 & 0 & 8.6 & 0 & 0 \\
\hline Naloxone treatment & $33^{*}$ & $4^{* *}$ & $2.5^{* *}$ & 4 & $30^{*}$ & 9.0 & $1.5^{*}$ & 0 \\
\hline
\end{tabular}

1) Class of behaviour: 1. normally occurring stereotypies

2. modulated "normal" stereotypies

3. new repetitive behaviours

4. drinking-directed behaviours

5. idling behaviours (sows could be alert, but inactive)

6. explorative behaviours directed to environmental objects

7. aggressive-like behaviours including escape attempts and "hiding" behaviours

8. other behaviours

2) Placebo (saline) was subcutaneously administered to tethered sows performing profound stereotypies and on the subsequent day the sows were treated with naloxone $(0.7-1.0 \mathrm{mg} / \mathrm{kg})$.

* different from placebo treatment $(* p<0.05, * * 00.01$, rank-sign test) 


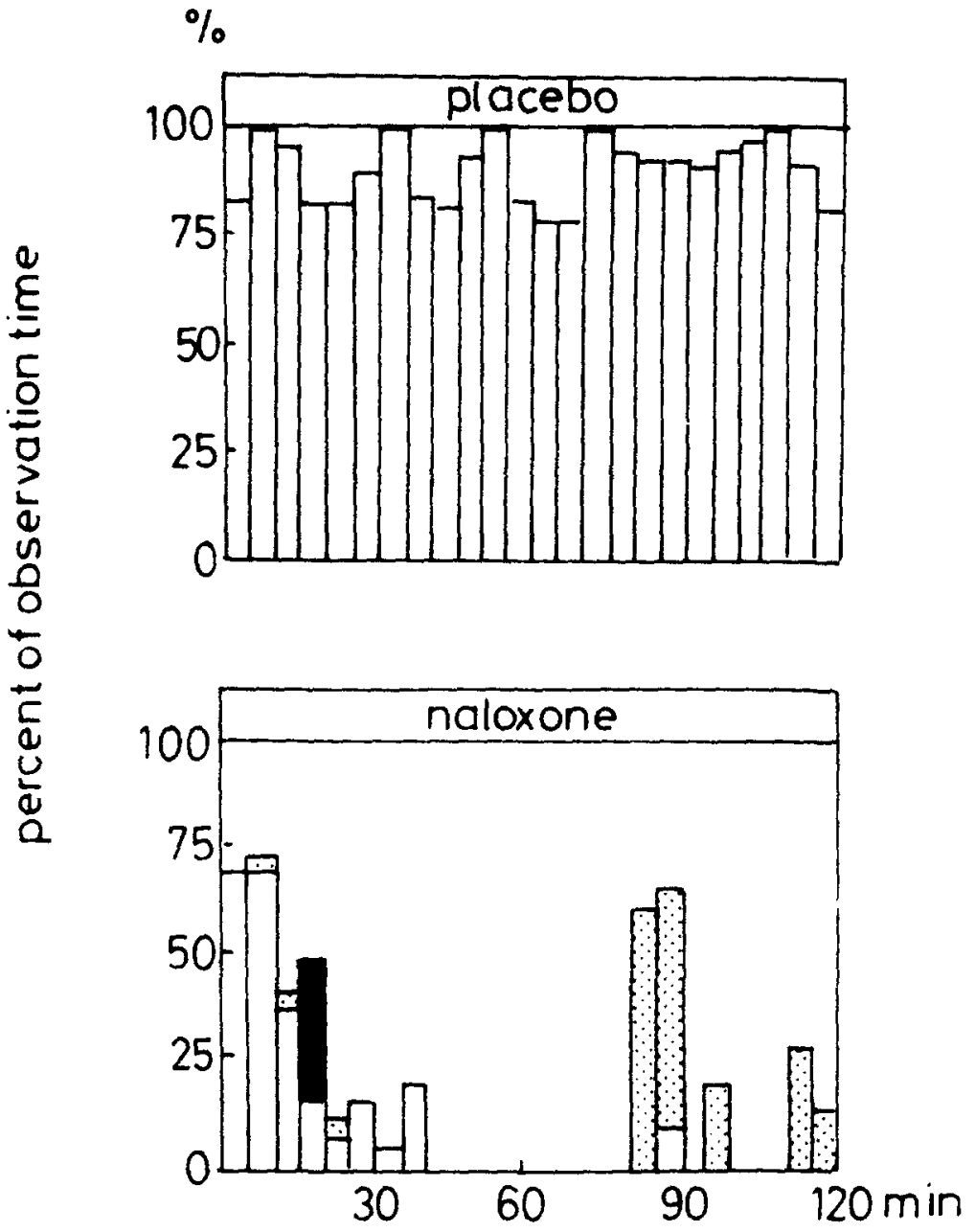

$\square$ normally occurring stereotypies (class 1 )

modified 'normal' stereotypies (class2)

0 new repetitive behaviours (class 3 )

FIG!KRE 1: An example of a profound effect of naloxone on stereotypies of tethered sows. The proportion of time that the sow performed stereotypies (per 5 min. period) is plotted versus the time after s.c. treatment with placebo : saline: or naloxore $0.96 \mathrm{mg}$ kj: 
The effect of naloxone usually started within 10-15 min. after injection and lasted about 2-3 hours. Modified "normal" stereotypies and some behaviours whichwere repetitive and had not previously been recorded for that particular sow were observed following naloxone. In the 2 hours following naloxone compared with saline, the sows were more inactive, but alert (class 5, table 1) and performed aggressive acts against the environment (attempts to attack neighbouring sows and to escape from the tether (class 7)). The effect of naloxone varied among the different sows. It was found that the degree of effect depended on the age of the stereotypies: the effect was stronger when the sow had performed her stereotypy for a shorter period of time.

Interestingly, the pattern of stereotypy performance was also changed in the days following naloxone treatment, as assessed in 3 sows. The sows showed less and, or modified stereotypies during the day time on the following days, and in addition performed these behaviours during the night time, a phenomenon which had never been observed in these sows before naloxone treatment. This aftereffect lasted at least 4-7 days.

The effect of naloxone appeared to be specific, since interfering with the normally occurring stereotypies in another way (e.g. by removal of the extra chain) did not result in changes in stereotypies on the following days. Moreover, naloxone did not alter the frequency and duration of explorative behaviours and behaviours directed to specific stimuli (straw, novel objects, soil) as was observed in a separate experiment involving 3 unrestrained sows.

\section{DISCUSSION}

The data show that naloxone treatment interrupts the stereotypies of tethered sows, suggesting that endorphins are implicated in these behaviours. Since naloxone specifically interferes with stereotypies and not with exploratory behaviours, it is assumed that the performance and maintenance of the stereotypies depend on the release of endorphins. These substances may calm the sows and decrease their aggressive-like behaviours evoked by the housing conditions. In this way the sows might cope with the conflict induced by the environment. Accordingly, aggression and other conflict behaviours which are characteristic for the initial period after being tethered (including escape attempts and "hiding") reappear after naloxone treatment. Thus, performing the seemingly purposeless stereotypies may function as an effective coping strategy for survival. That these behaviours are present during many hours a day may be related to the dependency-creating properties of endorphins (2) and therefore these stereotypies may be considered as an addirtive-like phenomenon. The endorphins may be especially implicated in the development of stereotypies, since older stereotypies seem to be less sensitive to naloxone treatment. Thus, in the course of the development of these behaviours, they may become less dependent of the original mechanisms, which may also hold for drug addictive behaviour (3).

\section{REFERENCES}

1. Cronin, G.M. and Wiepkema, P.R. (1984). An analysis of stereotyped behaviour in tethered sows. Ann. Rech. Vet. (in press).

2. Van Ree, J.M. (1979). Reinforcing stimulus properties of drugs. Neuropharmacology 18, 963-969.

3. Van Ree, J.M. (1980). Neurohypophysial hormones and addiction. In: De Wied, D. and Van Keep, P.A. (eds.) Hormones and the Brain. NTP Press L.tds., Lancaster. pp. $167-173$.

Accepted $28 / 7 / 85$ 